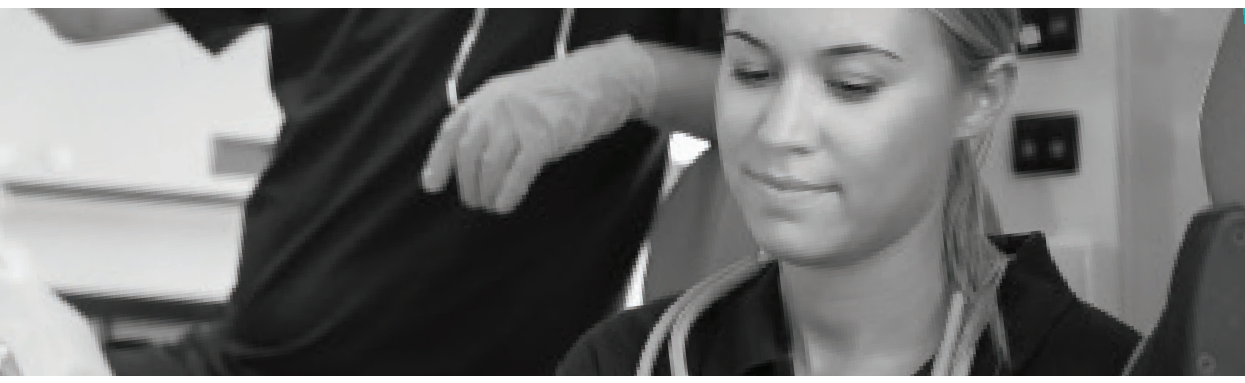

Volume 15 | Issue 1 | Article 4
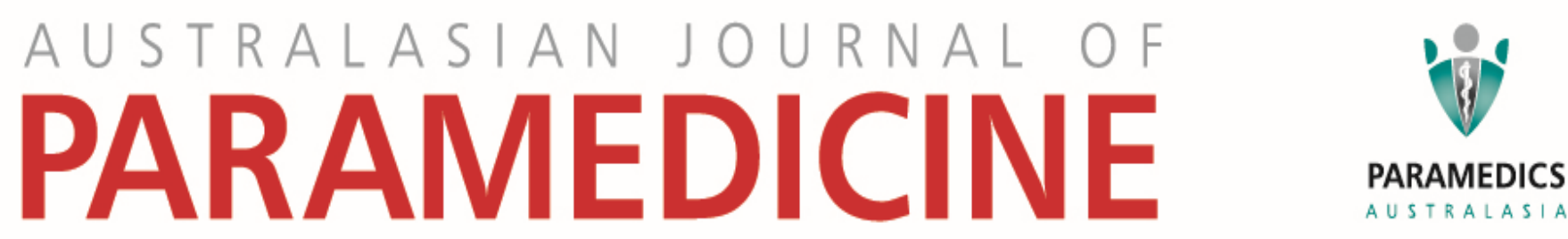

\title{
Pre-hospital advanced life support education - core components for pre-hospital professionals
}

\section{David Reid}

Edith Cowan University, Perth, Western Australia

Russell Jones

Edith Cowan University, Perth, Western Australia

Moira G Sim

Edith Cowan University, Perth, Western Australia 


\title{
Research
}

\section{Pre-hospital advanced life support education - core components for pre-hospital professionals}

\author{
David Reid MHM(Hons), GradCertHSM, BSci (Paramedic Science), Senior Lecturer ${ }^{1 ;}$ Russell Jones PhD, \\ BEd(Hons), DipEd, BSc, is Professor and Lead Emergency Services Research Group ${ }^{1}$; Moira G Sim MBBS(WA), \\ PGDipDrugAlcAbStud, FRACGP, FAChAM, is Professor and Executive Dean ${ }^{1}$
}

\author{
Affiliation: \\ ${ }^{1}$ School of Medical and Health Sciences, Edith Cowan University, Perth, Western Australia
}

\section{Abstract}

\section{Introduction}

There is a broad evidence base supporting advanced life support (ALS) education to health care professionals to be structured, realistic and inclusive of a range of human factors. However, published literature largely focusses on ALS education within institutional settings such as hospitals and associated health care facilities, with typically predictable human and material resources. The aim of this paper is to explore the literature on ALS education and report the results of an international survey undertaken to identify those educational elements which differ in the pre-hospital setting compared to the institutional setting. High quality education in pre-hospital resuscitation is important because it is critical that pre-hospital professionals provide high quality ALS to maximise the likelihood of patient survival.

\section{Methods}

MEDLINE was searched using MESH terms 'resuscitation', 'advanced life support' and 'prehospital' as core terms. Fifteen articles relating specifically to pre-hospital resuscitation and a further 91 articles on resuscitation education were reviewed. Articles ranged in publication year from 1987 to 2016.

An online survey on ALS education was developed and administered and attracted 177 responses from a range of health care professionals nationally and internationally. A total of 83 respondents completed the full survey.

\section{Results}

The literature identifies that best practice ALS education should (a) be taught in the environment in which ALS will be performed, (b) involve team members who are similar to those they will encounter while providing ALS, and (c) use equipment the provider uses in their workplace. Human factors, in particular leadership and communication, are important components of ALS education. However, results of the survey demonstrate that best practice is not well incorporated into pre-hospital ALS education.

\section{Conclusion}

To improve pre-hospital patient outcomes and maximise the chances of patient survival, ALS education to pre-hospital providers should incorporate the context of their workplace (that is, the environment in which they function), the team members they regularly work with and the equipment they use in the pre-hospital setting. Human factors, in particular leadership, should be considered as part of pre-hospital resuscitation education.

Keywords:

paramedic; resuscitation; pre-hospital; advanced life support; human factors

Corresponding Author: David Reid, d.reid@ecu.edu.au 


\section{Background}

The Australian Resuscitation Council (ARC) estimates that over 15,000 out-of-hospital cardiac arrests occur each year within Australia (1). During a 7 year period in Victoria (Australia), Ambulance Victoria attended 48,291 out-of-hospital cardiac arrests (2) and have identified that nationally there may be as many as 30,000 out-of-hospital cardiac arrests per annum (3). Cardiac arrests are defined as a 'combination of unresponsiveness and absent or abnormal breathing' (1).

The pre-hospital environment involves specific challenges including limited space, lighting and unpredictable bystanders (4). Pre-hospital providers may also experience the added challenge of moving a patient on a narrow stretcher and/ or in a moving vehicle often while performing resuscitation procedures. Yet the majority of training for managing cardiac arrests occurs in the hospital environment, which assumes access to space, equipment, lighting, fixed beds and other staff. These conditions are rarely all prevalent in the prehospital environment. Given these differences, the optimal training for management of cardiac arrests within the prehospital environment likely differs to that within a health care facility. Lyon and Sinclair (4) argue that 'a unique skill set is required to provide effective out-of-hospital resuscitation. A specifically designed national 'pre-hospital advanced life support course' would ensure the resuscitation standards of pre-hospital clinicians are maintained at the consistent high level required'. The number of out-of-hospital cardiac arrests in Australia, as well as the recognition that the pre-hospital environment presents unique challenges, provides compelling motivation to develop a specific pre-hospital ALS program. This paper discusses the literature on resuscitation education, and presents the results of a survey of pre-hospital providers on their experience of resuscitation education.

\section{Literature review}

MEDLINE was searched using MESH terms 'resuscitation', 'advanced life support' and 'prehospital' as core terms, limiting to articles published in the English language between 1987 and 2016. A total of 116 articles were identified and the abstracts of each article reviewed. Of these, 10 articles were found not to be relevant because they dealt with resuscitation of persons not in cardiac arrest, 15 articles related specifically to pre-hospital resuscitation and 91 articles related to resuscitation protocols, human factors or education.

The International Liaison Committee for Resuscitation (ILCOR) identified education interventions as one of the key attributes to target for improving outcomes from cardiopulmonary arrest (5). The European Resuscitation Council recognised the aim of educational interventions in resuscitation as to 'ensure that learners acquire and retain the skills and knowledge that will enable them to act correctly in actual cardiac arrests and improve patient outcomes' (6). The ARC endorsed these educational interventions (1).

From the literature reviewed, these key elements emerged as important in pre-hospital resuscitation education: mode of delivery, assessment, simulation and equipment, human factors and workplace implementation.

\section{Mode of delivery}

Resuscitation courses have traditionally been delivered face-to-face. However, there is an increased tendency toward blended learning due to the time poverty of clinicians, disparate locations and the desire for self-directed learning. This has led to the introduction of computer-aided learning as either an adjunct to, or replacement for, face-to-face training. There are advantages and disadvantages to blended learning combining face-to-face with other methods.

Computer-based training may improve resuscitation training effectiveness. One study of 28 second-year medical students found computer-based learning prepared students for practical exercises better than those students who were only trained using a traditional lecture and practice approach (7). The authors concluded computer training was useful because it assisted learners to memorise procedures before hands-on training sessions. Another study involved a randomised trial of 3732 health care professionals across 31 ALS centres in Australia and the United Kingdom. In this study, Perkins et al (8) evaluated the efficiency of cardiac arrest training between a blended interactive e-learning module followed by a one-day face-to-face course and a traditional two-day course delivered solely in a face-to-face course manner. The authors found both the blended and face-to-face groups performed equally well on a theory test. However, those students who studied via the blended route performed slightly worse in their end of course practical assessments than those who undertook the traditional course. After an undefined period of remedial teaching, following the end of course testing, the authors found that those participants who initially failed had improved enough to meet the minimum competency level. This suggests blended learning may need to include supplementary practical time for targeted students.

In another study, 113 final year medical students undertook either a face-to-face or multi-media ALS course (9). This study found there was no statistical difference between the two groups in the final written evaluation. However, the authors found a greater proportion of students who undertook the computer-aided learning required practical retesting to pass the course, and suggested that face-to-face teaching may result in improved 'first pass' results. The authors concluded the need for retesting was likely because students required additional familiarisation and training time on the manikins, which face-to-face learners had already experienced as part of their course. 


\section{Assessment}

There is broad agreement that competency assessment is an essential component of resuscitation education. Gjerra, Moller and Ostergaard (10) identified in their systematic review of trauma resuscitation that Kirkpatrick's (11) four-level model could be used to evaluate learning and included:

- reaction (participant satisfaction)

- learning (knowledge, skills and attitudes)

- behaviour (translation of learning to clinical setting)

- patient outcome.

Based on recommendations of international and Australian resuscitation bodies, most resuscitation courses involve both a theory and practical (generally competency-based) examination. Williams (6), in a literature review of resuscitation education, identified that even with specific practical tools there was variability in student results because examiners implemented such tools in a variable manner. Additionally, the literature review noted that a good result on a theoretical assessment did not consistently translate into the same level of competency in a practical scenario. Williams concluded that practical assessments should maximise reliability with a design that minimises variation in how candidates are assessed and marked in terms of their competency (6). The ability to design an assessment tool is challenging within resuscitation education because a large part of individual performance is based on human factors.

\section{Simulation and equipment}

The literature identified that education should be realistic and conducted in environments replicating real-world settings the student is likely to encounter $(12,13)$. One way of achieving this is through simulation. Providing simulation training in environments similar to that in which the students are expected to perform their skills enhances learning without endangering the lives of real patients. Bredmose et al (12) described their use of outdoor simulation for helicopter medical crews over a 2-year period. The authors identified the use of outdoor scenarios enabled crews to develop crew resource management skills, extrication and experience handling unsafe scenes and administering clinical care in uncontrolled environments.

In a 10-year review of resuscitation literature Kidd (13) concluded learner-centric techniques that combined theory and practical skills in resuscitation training were needed to optimise cardiac life support education and competencybased training. This author identified realism in training as vital. Further, scenarios needed to reflect the learners' own environment and be as realistic as possible. Confirming this finding, Lumsden (14), in a post-course review of a newborn life support course, found students welcomed simulation and rehearsal of skills in a realistic simulation environment before skill consolidation in a real clinical area.

Langdorf et al (15), in a study of 19 undergraduate medical students undertaking an intensive 20-hour advanced cardiac life support course, found the use of high fidelity resources improved learner performance and was preferred by students. Hoadley (16) and Aqel et al (17) in separate studies comparing groups of students training on high and low fidelity resources, found there was no statistical difference in terms of knowledge between groups of students trained on high or low fidelity manikins. Additionally, the authors found no difference between the groups for skills learned, satisfaction or self-confidence. These authors concluded that high-fidelity training did not produce higher gains than low-fidelity training. In another review Davis et al (18) concluded that highfidelity simulation was 'variable with respect to establishing significant improvements in knowledge and skills both gained and retained'. However, a review of the literature by Birkhoff and Donner (19) found high-fidelity simulation increased confidence and communication skills among medical and nursing staff and would be useful for paediatric advanced life support courses.

\section{Human factors}

Human factors, or non-technical skills, have become increasingly recognised in health care as important in prehospital resuscitation education, especially in situations which are high stress or high risk for the providers and/or patient. Gjerra, Moller and Ostergaard (10) defined non-technical skills as:

'The cognitive, social and personal resource skills that complement technical skills and contribute to safe and efficient task performance. Non-technical skills include situational awareness, decision-making, communication, teamwork, leadership and management of stress, fatigue and disturbances'.

The importance of human factors in resuscitation performance was underpinned in a study by von Wyl et al (20) in which 30 paramedics were rated for non-technical skills during a simulated resuscitation. A number of key human factors were identified as part of the literature review, and are discussed in the following sections:

- Leadership and co-ordination

- Interprofessional team working

- Communication.

\section{Leadership and co-ordination}

In a study of 81 nursing student scenarios by Husebo et al (21), the authors found leadership and co-ordination were critical during resuscitation. The authors identified coordination needed to be explicit and good co-ordination was supported by multiple modes of communication. If high quality co-ordination could be achieved, then team performance was likely to be higher. At times, paramedics will arrive on scene and have untrained persons performing basic life support who have received resuscitation advice over the phone from the ambulance co-ordination room. Paramedics also have a need to be able to lead and co-ordinate lay persons who have no experience in resuscitation. 


\section{Interprofessional teamwork}

In the pre-hospital setting paramedics often work in a small team of usually two paramedics. However, there may also be responders made up of representatives from other professions such as a nurse, physician or other allied health professionals. Therefore, while paramedics need to be able to work in a single discipline ambulance team, interprofessional teamwork is also important in the resuscitation setting.

Interprofessional team working is an accepted approach to teaching and work in the health care profession. In a study of 222 interprofessional fourth-year health care students using simulations by Dagnone et al (22) the authors found interprofessional training, with a particular focus on teamwork, improved the confidence of students. As a consequence of this interprofessional training, all participants reported a positive attitude to interprofessional working.

\section{Communication}

The importance of communication was highlighted in a systematic review of communication within trauma team training by Gjerra et al (10). The authors found communication errors occurred in $50 \%$ of the trauma resuscitation scenarios. The consequences of communication errors may include the incorrect administration of medications, or the inappropriate performance of procedures or technical skills. Resuscitation is a stressful clinical situation, and even more emotionally complex in the paediatric cardiac arrest situation. Birkhoff and Donner (19) reviewed the introduction of simulationbased training into a paediatric clinical setting. These authors identified communication errors occurred $100 \%$ of the time in mock paediatric cardiac arrests. They found that among student paramedics, nurses and physicians, the use of highfidelity manikins increased realism and increased student confidence and communication skills.

A year-long study reviewed the communication skills of 52 undergraduate and postgraduate nursing, respiratory and medical students at two separate points in time. This study conducted by Garbee et al (23) used a communication and teamwork skills framework to assess team performance. The study found communication was improved among a group of interdisciplinary students using crisis management principles and simulation. Further, the study found educational and professional structures were broken down and communication and teamwork skills improved.

\section{Workplace implementation}

Studies have identified that lessons and skills learned on a course are not consistently implemented in the workplace. Rasmussen and Dieckmann (24) and Currey and Considine (25) found, in semi-structured interviews with past participants of ALS courses, that although simulation-based training increased participants' self-confidence, there was an unwillingness to implement their skills back in the workplace. The authors found three key issues affected workplace implementation:

- Contextual adaptation - bringing new skills back into workplaces with pre-existing culture and practices

- Communities of practice - being integrated into their own workplace's ALS community and having the opportunity to 'prove' they were skilled at resuscitation

- Transfer - during actual resuscitation events transferring the leadership and knowledge to others who had not undergone the training.

In a study by Rasmussen and Tolsgaard (26) a survey of 249 former ALS course participants identified their ability to implement skills after their course was influenced by the level of teamwork and their co-workers' skills in the workplace.

Respondents were asked about co-worker behaviour, communication and teamwork.

Based on the results of the literature review it was clear that best practice ALS education should (a) be taught in the environment in which ALS will be performed, (b) involve team members who are similar to those they will encounter while providing ALS, and (c) use equipment the provider uses in their workplace. Human factors, in particular leadership and communication, are important components of ALS education. An online survey was developed and administered to explore pre-hospital practitioners' experience of ALS education.

\section{Methods}

An online survey on ALS education was developed to examine:

- the experiences of pre-hospital providers in resuscitation education

- comparisons of education scenarios, equipment and human factors to respondents actual resuscitation experience

- workplace implementation of the providers' resuscitation skill-set.

Survey validity was tested by administering the questions to a small cohort of six experienced pre-hospital health care providers who were also university lecturers. A briefing on the aims of the survey in the context of the overall research was provided before the survey was administered for completion and comment. Feedback was obtained from the providers on the survey content, question scales and whether the questions accurately captured their views on resuscitation education and actual experience of resuscitation. Following amendments, the survey was readministered to a small cohort of the same prehospital professionals and further feedback gathered. 
The survey was administered between 27 April 2015 and 16 July 2015 to volunteer and paid health care professionals such as medical practitioners, nurses, paramedics and first responders who worked in the pre-hospital setting. The survey contained quantitative and qualitative elements. The survey consisted of 22 questions in three sections: a) the first 10 questions on resuscitation education undertaken, b) five questions on actual resuscitations undertaken, and c) six demographic questions. One question was open-ended and sought final comments from participants on pre-hospital resuscitation in general.

The survey was administered online via SurveyMonkey® software and promoted using a snowball technique (27) through pre-hospital contacts of university academics, personnel in clinical settings, social media including Linkedln and the paramedic professional representative body, Paramedics Australasia. The survey was approved by the host institution's Human Research Ethics Committee.

\section{Results}

The online survey attracted 177 responses from a range of health care professionals internationally from countries including Australia, New Zealand, the United Kingdom and the United States of America (USA). All 177 respondents (100\%) completed Section A on resuscitation education undertaken, $85(48 \%)$ responded to Section B pertaining to actual resuscitations undertaken, and $83(47 \%)$ responded to Section $\mathrm{C}$ about their demographic information. The 92 respondents who did not answer questions in Section B may not have been involved in actual resuscitations or preferred not to answer questions about this topic.

As the questions about personal characteristics of the participants were presented at the end of the survey, demographic information is only available for the $47 \%$ who completed the entire survey. Of these, the majority of respondents (31\%) were qualified ambulance paramedics or critical care paramedics (22\%). Other respondents included emergency medical technicians and first respondents (7\%), ambulance officers (paramedics in-training) (10\%), medical practitioners $(6 \%)$, educators $(7 \%)$, registered nurses $(4 \%)$, first aiders $(2 \%)$ and others ( $11 \%)$. In all, $62 \%$ of respondents were employed by pre-hospital state emergency ambulance services, with $22 \%$ of respondents working for volunteer organisations.

\section{Course factors}

All respondents to the survey reported their resuscitation course was taught face-to-face. Seventy-four percent of respondents undertook theoretical reading before the course; $17 \%$ of respondents indicated they did not complete any readings either before or after their course and $10 \%$ reported they completed reading after the course. Eighty percent of respondents strongly agreed or agreed that theory readings helped them understand the process of resuscitation; $73 \%$ of respondents strongly agreed or agreed that theory readings were applicable to their area of clinical practice.

\section{Simulation}

Ninety-five percent of respondents identified that their course included simulation (Table 1). The level of simulation fidelity was not specified.

Sixty-eight percent of respondents reported they undertook training scenarios in a pre-hospital environment; $29 \%$ of respondents indicated scenarios took place in an emergency department, $28 \%$ in a hospital ward and $26 \%$ in a home situation such as a kitchen or lounge room. Twelve percent of respondents indicated simulation was held in a health clinic environment and a further $36 \%$ in a range of other areas including care facilities and operating theatres. Respondents were able to choose more than one answer and it is likely that most undertook scenarios in more than one location.

Only $35 \%$ of respondents to the survey reported the location of their real resuscitations was 'almost identical' or 'very similar' to their training location. Twenty-eight percent of respondents identified that environmental factors (eg. heating, lighting) were 'almost identical' or 'very similar' to their training environment. Less than half the respondents (46\%) indicated that actual patient movement and patient extrication was similar to their training (Figure 1).

Table 1. Resuscitation course simulation scenarios

\begin{tabular}{|l|c|c|c|}
\hline \multicolumn{1}{|c|}{ Course scenarios } & Yes & No & $\begin{array}{c}\text { Total } \\
\text { (n=150) }\end{array}$ \\
\hline $\begin{array}{l}\text { The resuscitation course I attended included practical resuscitation } \\
\text { scenarios }\end{array}$ & $95.27 \%$ & $4.73 \%$ & $100.00 \%$ \\
\hline $\begin{array}{l}\text { The resuscitation course I attended incorporated multi-disciplinary teams } \\
\text { during the scenarios (eg. doctors, nurses, paramedics, allied health) }\end{array}$ & $39.33 \%$ & $60.67 \%$ & $100.00 \%$ \\
\hline $\begin{array}{l}\text { Varying numbers of team members were included in the scenarios (eg. one } \\
\text { scenario having three team members and another having six) }\end{array}$ & $52.67 \%$ & $47.33 \%$ & $100.00 \%$ \\
\hline
\end{tabular}


Environment in which the resuscitation occurred

(e.g., lighting, heating, indoor vs. outdoor etc.)

Location in which the resuscitation occurred

Patient movement/extrication during resuscitation
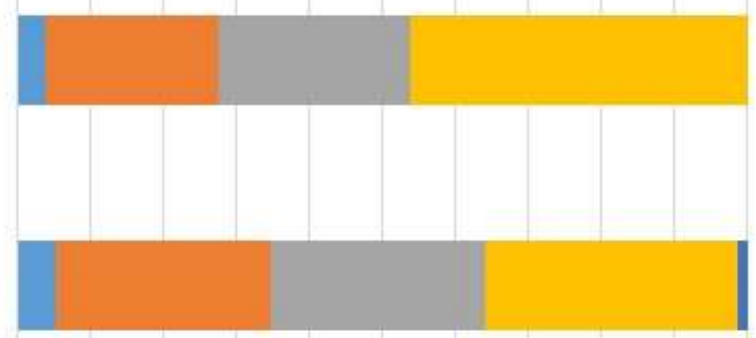

$\begin{array}{lllllllllll}0 \% & 10 \% & 20 \% & 30 \% & 40 \% & 50 \% & 60 \% & 70 \% & 80 \% & 90 \% & 100 \%\end{array}$

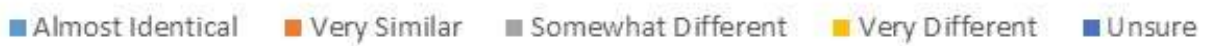

Figure 1. Real resuscitation environment in relation to training environment

A number of respondents to the survey commented on the simulation aspects of their resuscitation course and provided additional observations on improvement opportunities: 'Most resuscitations as evidenced occur out of hospital with perhaps one or two professional staff + - lay persons on scene. However every BLS, ALS course I have ever attended have only concentrated on the in-hospital setting which is inherently different in nearly every aspect.'

'Having taught and been taught both in and pre-hospital as both a paramedic and now a doctor it is my opinion that the pre-hospital teaching provides a more flexible approach to resuscitation which can be adapted to hospital, the converse is not as true.'

'I am yet to find a paramedic specific ALS course, which incorporates two provider ALS with a time delay before two to three more responders arrive, such as in a real pre-hospital resuscitation. Paramedic ALS courses should include patient treatment and movement in awkward areas/rooms/lighting to simulate real cases; a time delay before more responders arrive to help, and scenarios where paramedics have to decide to stop resuscitation efforts.'

'Resuscitation scenarios should closely reflect actual practice. Patients do not go into cardiac arrest on tables in well-lit rooms. Scenarios that contain loud noise, lots of bystanders, low lighting etc.'

\section{Equipment used}

Equipment used during resuscitation can include defibrillators, equipment for oxygenation and equipment for airway and circulation management. Seventy-six percent of respondents to the survey reported the equipment used in their training was 'almost identical' or 'very similar' to that used in their real resuscitations, while $24 \%$ indicated the equipment was 'somewhat' or 'very' different. Seventy-nine percent of respondents identified that the medications used were 'almost identical' or 'very similar', while $17 \%$ indicated that medications used were 'somewhat' or 'very' different.

\section{Human factors}

Sixty-three percent of respondents reported leadership during their actual resuscitations was almost identical or very similar to that experienced on their course; $49 \%$ of respondents 'strongly agreed' or 'agreed' that the role of team members in the real resuscitations they experienced were the same or similar to the roles involved in their training. For example, simulation training involved doctors and nursing staff, and in real life the same types of staff were present. Forty-one percent of respondents identified that the same number of team members were present in real resuscitations as were included in their training (Table 2).

Teamwork attracted the most comments from respondents to the survey:

'ALS courses should undertake simulation more realistically, with more time spent on logistics within emergency situations, such as the pit crew approach which is commonly used pre-hospitally (sic) but unheard of in-hospital. Also better simulation with differing team members and dynamics, instead of usual approach of one team leader being assessed and other staff can not show any initiative/input, which is unlike real life scenarios.'

'I have been teaching ACLS and PALS for some time now and have always commented that the 'team learning approach' is a bit odd in that we never practise with our actual teams.' 
Reid: Pre-hospital advanced life support education

Australasian Journal of Paramedicine: 2018;15(1)

Table 2. Resuscitation teams in actual resuscitations compared to training environment

\begin{tabular}{|l|c|c|c|c|c|c|}
\hline \multicolumn{1}{|c|}{ Answer options } & $\begin{array}{c}\text { Strongly } \\
\text { agree }\end{array}$ & Agree & Neutral & Disagree & $\begin{array}{c}\text { Stongly } \\
\text { disagree }\end{array}$ & $\begin{array}{c}\text { Total } \\
\text { (n=81) }\end{array}$ \\
\hline $\begin{array}{l}\text { The resuscitation(s) I have } \\
\text { been involved in had the } \\
\text { same or very similar team } \\
\text { members by role as were } \\
\text { involved on the course (eg. } \\
\text { team of doctors and nurses } \\
\text { on course and same during } \\
\text { actual resuscitation or } \\
\text { nurses/paramedics on course } \\
\text { and same during the real } \\
\text { resuscitation) }\end{array}$ & $16.05 \%$ & $30.86 \%$ & $7.41 \%$ & $28.40 \%$ & $17.28 \%$ & $100.00 \%$ \\
\hline $\begin{array}{l}\text { The resuscitation(s) I have } \\
\text { been involved in had the } \\
\text { same or similar numbers } \\
\text { of team members in the } \\
\text { resuscitation (eg. six team } \\
\text { members in course and six } \\
\text { during real resuscitation) }\end{array}$ & $16.05 \%$ & $33.33 \%$ & $8.64 \%$ & $27.16 \%$ & $14.81 \%$ & $100.00 \%$ \\
\hline
\end{tabular}

The resuscitation course prepared me for the clinical setting in which I carry out resuscitations (e.g., ward, $E D$, operating theatre, prehospital)

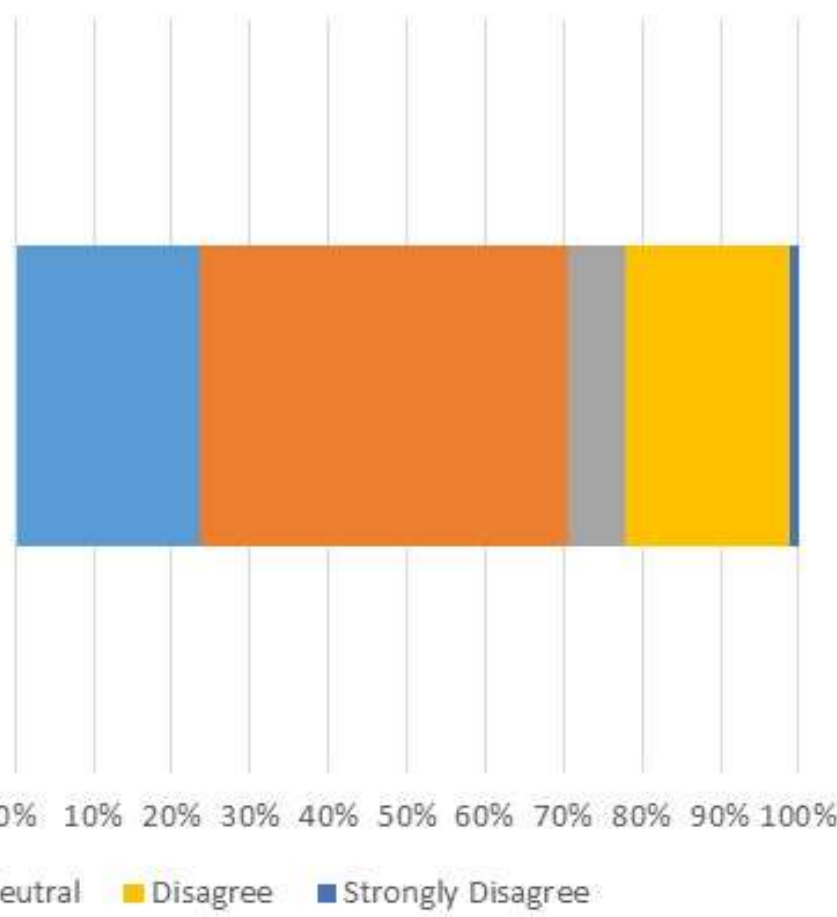

Figure 2. Preparation of course for real resuscitations 


\section{Workplace implementation}

Thirty-five percent of respondents reported the location of their real resuscitations was 'almost identical' or 'very similar' to their training; $28 \%$ of respondents identified environmental factors (eg. heating, lighting) were 'almost identical' or 'very similar' to their training environment; $70 \%$ of respondents strongly agreed or agreed their resuscitation course prepared them for the clinical setting in which they carried out resuscitation (Figure 2).

Survey participants, the majority of whom were paramedics, reported that all pre-hospital resuscitation courses they attended were held face-to-face, and in some cases involved some pre-reading. Seventeen percent of respondents identified they did no reading either before or after the course. Respondents had noted changes in training over the years and made the following general comments about training: 'From the pre-hospital setting perspective there does not seem to be enough emphasis on evidence based medicine, although for the last 2-4 years in the USA there has been a big push to do so.'

'I believe training has got better from when I started in the ambulance service but there is still a long way to go.'

From the free-text area, a number of respondents identified areas where they would like a pre-hospital course to focus:

- pre-hospital scene control

- patient assessment

- working as multi-disciplinary team with physicians as team leaders/members

- extraction

- family counselling post resuscitation.

\section{Discussion}

The literature on resuscitation identified that the pre-hospital environment presents unique challenges to those performing resuscitation (4). The core components which contribute to high quality resuscitation education are also well identified in the literature and include the mode of delivery, use of simulation, equipment and competency assessment in order to improve patient outcomes (6). Other key areas identified in the literature were the role of human factors in resuscitation and implementation of the knowledge and skills back in the workplace. However, there is a gap in education standards for pre-hospital health care providers, and it is in that context in which the review and discussion took place.

\section{Course factors}

The literature identified that online learning was no worse than face-to-face course delivery in theoretical knowledge gain in resuscitation $(5,7)$. The survey completed as part of this study found although $84 \%$ of respondents undertook pre- or postcourse reading, the teaching was all undertaken face-to-face. This result may be a consequence of the snowball technique used to identify survey respondents, who were primarily paramedics, and requires further investigation to determine if pre-hospital health care professionals are more likely to participate in face-to-face resuscitation courses than other health care professionals.

Simulation education should be realistic and held in environments not dissimilar to where the student is likely to encounter real patients $(12,13)$. The literature identified that simulation ensures skills can be learned in a controlled environment and mistakes made without endangering patient welfare. The literature also identified realism in simulation, which includes reflecting the teams and equipment used in the participants' usual work environment, is an important aspect of training (12-14). The survey completed as part of this research identified that, commensurate with the literature, simulation was used in all courses. However, over $60 \%$ of respondents indicated their training did not include multidisciplinary teams, and almost $50 \%$ of respondents indicated their scenarios did not include varying numbers of team members as would commonly be found in the pre-hospital environment. Almost a third of respondents to the survey indicated their scenarios did not include simulation in a pre-hospital environment. Almost $65 \%$ of respondents indicated the location in which they have carried out real resuscitations differed from the environment in which they undertook simulation, aligning with recommendations from the existing literature. These results indicate there is significant room for improvement in conducting targeted pre-hospital resuscitation training in the environment in which participants work and with the teams they work.

There was mixed evidence on the benefits and disadvantages of high and low fidelity in simulation $(16,18,19)$. Although studies found that there was no statistical difference in terms of the skills learned, some identified that high-fidelity simulation increased confidence and student communication skills. The pre-hospital resuscitation survey conducted did not focus on the use of high or low fidelity simulation and this is an area for further research. However, over $75 \%$ of respondents to the survey indicated that the equipment they used in training was similar to that used in their workplace, and almost $80 \%$ indicated that the medications used were similar to those used in their workplace.

The peak resuscitation bodies ARC and ILCOR identified that competency assessments were an essential component of resuscitation education (10). Supporting this literature, the results of the pre-hospital health care provider survey indicated that all respondents were examined using competency assessment processes.

\section{Human factors}

Non-technical skills in resuscitation, including leadership and co-ordination and communication, have been identified in the literature as taking increasing prominence $(10,20)$. These authors found a positive correlation between technical and behavioural performance and human factors including leadership, co-ordination and communication. 
Interprofessional teamwork was highlighted in the literature as contributing to improved confidence and co-ordination in resuscitation (22). Fifty percent of survey respondents indicated the profession of team members differed between training and actual resuscitations and $59 \%$ of respondents indicated the number of team members differed between training and actual resuscitations. The results of the survey indicate there are improvement opportunities in ensuring pre-hospital health care professionals are trained to work with team members who will be present during their actual resuscitations.

Communication, including leadership and co-ordination, was another critical human factor element identified in the literature. One study of trauma teams' communication found errors occurred in $50 \%$ of resuscitation training scenarios (10). The use of crisis management principles was found to break down structures and improve behaviour and teamwork skills (23). Results of the survey conducted as part of this research found $37 \%$ of respondents experienced different leadership during actual resuscitations experienced compared with their training. This result indicates there is room for improvement in terms of preparing pre-hospital professionals for leadership roles in resuscitation.

\section{Workplace implementation}

While education is important, unless the skills acquired during education can be implemented back in the workplace the training will not have any practical impact in real resuscitations. The literature identified implementation in the workplace as challenging $(25,26)$. Particular areas identified in the literature as limiting implementation in the workplace were contextual adaptation, communities of practice and transfer of skills into the real environment (25). While $70 \%$ of respondents indicated that training prepared them for the clinical setting in which they carried out actual resuscitation, almost a third indicated their training did not prepare them. Sixty-five percent of respondents indicated the location of real resuscitation was different to their training, with $72 \%$ of respondents indicating that environmental factors such as lighting were different. The results of the survey indicate that pre-hospital health care professionals could be more consistently trained in their working environment, in order to better prepare them for actual practice.

\section{Conclusion}

To improve patient outcomes and maximise the chances of patient survival it is important pre-hospital providers be taught ALS in the environment in which they operate with team members they regularly work with and using equipment they will use in the pre-hospital setting. Human factors, in particular leadership and communication, should be considered as part of pre-hospital resuscitation education.

Results of the survey demonstrate there is room for improvement in pre-hospital resuscitation education. Particular areas for improvement include course participants training with the staff with whom they work, educating team members with the number of clinicians and bystanders they will encounter in the field, and introducing the unique characteristics of pre-hospital resuscitation such as training outdoors and preparation for transport.

\section{Limitations}

This research has a number of limitations which may impact generalisability. The sample size is modest in relation to Australian and global pre-hospital health care professionals. Additionally, not all providers completed the full survey and it is assumed this is because not all respondents have attended an out-of-hospital cardiac arrest. This limits applicability across all pre-hospital providers. As a base-line survey for pre-hospital professionals on resuscitation not all aspects of education were examined. Areas of education which could be examined in-depth in future studies could include whether respondents were tested on their pre-course reading and simulation fidelity. The survey is based on the views of individuals and the opinion of any one participant may not be able to be generalised to the population. The total number of pre-hospital providers is unknown, so it is not possible to statistically determine whether those who responded to the survey are a representative sample of pre-hospital health care providers. There is a lack of evidence on which elements of resuscitation training most impact actual performance in the pre-hospital field and the literature identified only those elements which impact hospital-based resuscitation. The snowball technique used to collect data for this study may have resulted in a sample that is unrepresentative of the population. The survey was taken at a point in time, and the changing views of respondents have not been captured. Over time the responses of individuals may change as they reflect on the educational experience or undertake actual resuscitations.

\section{Acknowledgements}

The authors wish to thank all the providers who responded to the survey and Mr Mike Gale from the Australian Resuscitation Council who provided advice to the literature review.

\section{Conflict of interest}

The authors declare no competing interests. The authors of this paper have completed the ICMJE conflict of interest statement. 


\section{References}

1. Australian Resuscitation Council. Cardiac arrest - An introduction. Undated. Available at: https://resus.org.au/ guidelines/

2. Gale M, Grantham H, Morley P, Parr M. Advanced life support level 2. Perth: Australian Resuscitation Council; 2016.

3. Lijovic M. Victorian Ambulance cardiac arrest registry. Annual report 2014-15. Melbourne: Victorian Ambulance; 2016.

4. Lyon RM, Sinclair N. National standards in prehospital resuscitation training are required. Emerg Med $\mathrm{J}$ 2012;29:602.

5. Perkins G, Fullerton JN, Davis-Gomez N, et al. The effect of pre-course e-learning prior to advanced life support training: a randomised controlled trial. Resuscitation 2010;81:877-81.

6. Williams NM. Advanced life support training and assessment: a literature review. Australas Emerg Nurs J 2011;14:240-5.

7. Bonnetain E, Boucheix J, Hamet M, Freysz M. Benefits of computer screen-based simulation in learning cardiac arrest procedures. Med Educ 2010;44:716-22.

8. Perkins GD, Kimani PK, Bullock I, et al. Improving the efficiency of advanced life support training: a randomized, controlled trial. Ann Intern Med 2012;157:19-28.

9. Christenson J, Parrish K, Barabé S, et al. A comparison of multimedia and standard advanced cardiac life support learning. Acad Emerg Med 1998;5:702-8.

10. Gjerra K, Moller TP, Ostergaard D. Efficacy of simulationbased trauma team training of non-technical skills. A systematic review. Acta Anaesthesiol Scand 2014;58:77587.

11. Kirkpatrick D, Kirkpatrick J. Evaluating training programs the four levels. Berrett-Koehler; 2006.

12. Bredmose PP, Habig K, Davies G, Grier G, Lockey DJ. Scenario based outdoor simulation in pre-hospital trauma care using a simple mannequin model. Scand J Trauma Resusc Emerg Med 2010;18:13.

13. Kidd T, Kendall S. Review of effective advanced cardiac life support training using experiential learning. J Clin Nurs 2007;16:58-66.

14.Lumsden H. Newborn life support course: training students. Br J Midwifery 2008;16:717-21.
15. Langdorf MI, Strom SL, Yang L, et al. High-Fidelity Simulation Enhances ACLS Training. Teach Learn Med 2014;26:266-73.

16. Hoadley TA. Learning advanced cardiac life support: a comparison study of the effects of low- and high-fidelity simulation. Nurs Educ Perspect 2009;30:91-5.

17. Aqel AA, Ahmad MM. High-fidelity simulation effects on CPR knowledge, skills, acquisition, and retention in nursing students. Worldviews Evid Based Nurs 2014;11:394-400.

18. Davis LE, Storjohann TD, Spiegel JJ, Beiber KM, Barletta JF. High-fidelity simulation for advanced cardiac life support training. Am J Pharm Educ 2013;77:59.

19. Birkhoff SD, Donner C. Enhancing pediatric clinical competency with high-fidelity simulation. J Contin Educ Nurs 2010;41:418-23.

20. Von Wyl T, Zuercher M, Amsler F, Walter B, Ummenhofer W. Technical and non-technical skills can be reliably assessed during paramedic simulation training. Acta Anaesthesiol Scand 2009;53:121-7.

21. Husebø SE, Rystedt H, Friberg F. Educating for teamwork - nursing students' coordination in simulated cardiac arrest situations. J Adv Nurs 2011;67:2239-55.

22. Dagnone JD, McGraw RC, Pulling CA, Patteson AK. Interprofessional resuscitation rounds: a teamwork approach to ACLS education. Med Teach 2008;30:e49-54.

23. Garbee DD, Paige J, Barrier K, et al. Interprofessional teamwork among students in simulated codes: a quasiexperimental study. Nurs Educ Perspect 2013;34:339-44.

24. Rasmussen M, Dieckmann P, Barry Issenberg S, et al. Longterm intended and unintended experiences after Advanced Life Support training. Resuscitation 2013;84:373-7.

25. Currey J, Considine J, Allen J. Learner perceptions and reflections after simulation-based advanced life support training. Aust Crit Care 2014;27:103-5.

26. Rasmussen M, Tolsgaard MG, Dieckmann P, et al. Factors relating to the perceived management of emergency situations: a survey of former advanced life support course participants' clinical experiences. Resuscitation 2014;85:1726-31.

27. Goodman LA. Snowball sampling. Annals of Mathematical Statistics 1961;32:148-70. 